\title{
Nrf2 Antioxidant Pathway Suppresses Numb-mediated Epithelial-mesenchymal Transition during Pulmonary Fibrosis
}

\author{
Zhihui Zhang ${ }^{1}$, Jiao $\mathrm{Qu}^{2,3}$, Cheng Zheng ${ }^{1}$, Panpan Zhang ${ }^{3}$, Wencheng Zhou ${ }^{4}$, Wenhui Cui ${ }^{4}$, \\ Xiaoting $\mathrm{Mo}^{4}$, Liucheng $\mathrm{Li}^{1}$, Liang $\mathrm{Xu}^{1}$, Jian $\mathrm{Gao}^{1}$ \\ ${ }^{I}$ The First Affiliated Hospital of Anhui Medical University, China, ${ }^{2}$ The Second Affiliated Hospital of Dalian Medical \\ University, China, ${ }^{3}$ School of Pharmacy, Dalian Medical University, China, ${ }^{4}$ School of Pharmacy, Anhui Medical \\ University, China
}

\section{Abstract}

Background: Epithelial mesenchymal transition (EMT) is a key progression that promotes pulmonary fibrosis (PF). Numb, a phosphotyrosine-binding domain (PTB) protein, is implicated with EMT. Nuclear factor erythroid 2 related factor 2 (Nrf2) and its downstream proteins, heme oxygenase-1 (HO-1) and $\mathrm{NAD}(\mathrm{P}) \mathrm{H}$ : quinone oxidoreductase 1 (NQO1), constitute an important pathway of antioxidant defense signal for protecting against PF. It remains elusive whether Nrf2 antioxidant pathway and Numb have a potential relationship in EMT-mediated PF.

Method: Building bleomycin (BLM)-induced PF in Nrf2-knockout (Nrf2-/-) and wild-type (WT) mice to observe the effects of Nrf2 pathway and Numb by Western blot and immunohistochemistry. Meanwhile, rat type II alveolar epithelial cells line (RLE-6TN) and human epithelial cells line (A549) were both treated with an Nrf2 activator sulforaphane (SFN), or transfected siRNAs of Nrf2 and Numb to unravel roles of Nrf2 pathway, Numb and the link between them in transforming growth factor\&beta1 (TGF\&beta1)-induced EMT.

Result: BLM-induced lung fibrosis were more severe in Nrf2-/- mice compared to WT mice with reduced expressions of HO-1 and NQO1. Numb was enhanced with down-regulated expressions of Nrf2 in BLM groups and further increased in Nrf2-/- groups. In vitro, given exogenous TGF\&beta1 on RLE-6TN and A549 up-regulated Numb expression with downregulations of Nrf2 and its target proteins HO-1 and NQO1. Transfected with Nrf2 and Numb siRNAs further aggravated and relieved the progression of EMT. Inversely, activating Nrf2 pathway by SFN reduced the expression of Numb and alleviated TGF\&beta1-induced EMT. Interestingly, Numb deficiency by siRNA relieved the protection of activating Nrf2 against EMT.

Conclusion: Activating Nrf2 antioxidant pathway suppresses EMT during PF via inhibiting the abnormal expression of Numb. These findings provide insight into PF pathogenesis and a basis for novel treatment approaches.

Corresponding to:

Prof. Jian Gao, $\mathrm{PhD}$,

The First Affiliated Hospital of Anhui Medical University,

Jixi Road 218,

Hefei, 230022, China.

Tel:+86-551-62922423

Fax:+86-551-62922423

Email: gaojianayfy@163.com. 